\title{
QUADRICEPS WASTING IN RELATION TO INJURIES INVOLVING THE ANKLE JOINT
}

\author{
A. BARRANES, M.S.R.G., B.A.S.M., M.R.S.H. \\ Medical Rehabilitation Officer, Albert Dock Orthopaedic Hospital.
}

The importance of quadriceps and gluteal exercises for injuries involving the ankle joint, have, in the past, been overshadowed by the primary aims of mobilising and redeveloping the ankle and foot movements, muscles of the lower leg, and longitudinal and transverse arches of the foot.

Of 40 cases seen within $48 \mathrm{hrs}$. of injury, who were capable of weight-bearing and placed on immediate re-educated walking, ankle and quadriceps exercises:-

Two weeks after injury:-

11 showed wasting of $1 / 2$ " thigh circumference

29 showed wasting of $1 / 2-3 / 4$ " thigh circumference.

This measurement was taken 4" above the upper pole of the patella. This loss recovered once full mobility was achieved, although this recovery time varied considerably in individuals. These observations were made over a period of two years. Many cases could not be included due to various lengths of time before their appearance at the clinics although the wastage was obvious.

The functional and mechanical action that takes place when there is restricted ankle movement consists of abducting and externally rotating the leg with the knee joint extended, using the heel as a point of pivot.

There are two reasons for this:

Firstly, this action allows walking to take place with the minimum of movement at the injured joint, thereby preventing unnecessary pain and discomfort. It is basically a natural protective action.

Secondly, it allows long strides to be taken with the good limb, and, by carrying the injured limb through in the abducted position, it enables a fairly fast form of ambulation.

It is for these reasons that careful attention should be given to re-education in the correct walking technique.

It cannot be over-emphasized that the first two lessons of re-education in walking must be given under careful supervision, and with appropriate explanation. Once the "short step" walk has been mastered, improvement will become fairly rapid, giving immense confidence. It should be remembered, that the patient must be made aware of the fact, that, as early movement is gained, there will be some pain and swelling, and that when resting the limb, it should be elevated. Also, that as the movement increases, so the pain will diminish.
Periodic supervision of correct walking is usually necessary for at least two weeks, by which time, the patient should be walking correctly with short paces. It will be found that progress will be gained by teaching the patient to walk with the feet approximately four inches apart, with the toes pointing to the front. This gives a squarer base for stability, and to walk in this manner, true ankle movement is required.

Having taught the patient the correct "short step" walk, we are faced with two groups of muscles, the quadriceps and the gluteus maximus, being restricted in their mechanical functions, because the limited ankle movement will cause the patient to walk with the knee joint slightly flexed. This knee flexion commences with the beginning of the stride, and is continued throughout the whole movement, inevitably causing a relaxation of the hamstrings and wasting of the vastus medialis muscle, with a potential contracture of the posterior part of the capsule of the knee joint. This in itself gives rise to a feeling of weakness and instability. In a similar manner, because of the "short step" walk that the patient has been taught, the gluteus maximus is not fully required to extend the thigh at the hip joint, thereby restricting the range of the muscle function.

Static quadriceps and gluteal contractions should be commenced as early as possible, and, after correct walking has been obtained with lessening of painful movement, it is then advisable to advance to graduated resisted exercises. It should be remembered that, if the patient starts to feel unstable at the knee joint, combined with the already conscious knowledge of the injured ankle, he will immediately restrict his activities, thereby retarding his own recovery still further.

In bilateral cases there will be found an even greater tendancy to walk with the knees flexed. It may be doubted at first that a wasting of quadriceps and gluteus maximus occurs in injuries involving the ankle joint, but if the two limbs are compared, it will be found that, in seven cases out of ten, the wasting is easily seen, especially at the site of vastus medialis. This wasting is even more pronounced in cases of fracture of the os calcis, where walking is usually very restricted in the early days of treatment, and this especially so in bilateral fractures although there are, of course, other factors to be taken into consideration in this type of case. 